\title{
The Effect of Adrenalectomy on Leptin Levels and Some Metabolic Parameters in Rats with Diet-Induced Obesity
}

\author{
Adnan Yilmaz, ${ }^{a}$ Halis Suleyman, ${ }^{b}$ Zuhal Umudum, ${ }^{a}$ and Yasar Nuri Sahin ${ }^{*, c}$ \\ ${ }^{a}$ Department of Biochemistry, Faculty of Medicine, University of Ataturk; TR25240, Erzurum, Turkey: ${ }^{b}$ Department of \\ Pharmacology, Faculty of Medicine, University of Ataturk; TR25240, Erzurum, Turkey: and ${ }^{c}$ Department of Biochemistry, \\ Faculty of Medicine, University of Ataturk; TR25240, Erzurum, Turkey. \\ Received November 19, 2001; accepted February 5, 2002
}

Recently, there has been many investigations on the relationship between leptin and obesity, which is the main health problem in developed countries. In some reports, it has been claimed that the adrenalectomy has lead to weight loss and thus prevented obesity induced in rodents in various ways. It has also been accepted that diet-induced obesity in animals is very similar to obesity in humans beings. In this study, obesity has been developed with high-calorie diet given for 8 weeks in Sprague-Dawley rats. Then, it has been investigated how leptin and some metabolic parameters change in blood samples obtained from rats $\mathbf{1 5} \mathrm{d}$ after adrenalectomy. Leptin levels was determined with Radıo Immun Assay (RIA, Linco Research Co ${ }^{\circledR}$ ) method. Our study showed that, there were statistically significant increases in leptin $(p<0.001)$, glucose $(p<0.05)$, triglyceride $(p<0.01)$ levels in diet-induced obese rats $(n=19)$ when compared with the findings of control rats, lean ones $(n=16)$, $($ Tables 3,4$)$. Adrenalectomy led to decreased serum leptin $(p<0.001)$ and triglyceride $(p<0.01)$ levels both in the obese and lean rats (Table 5). As a conclusion, it could be claimed that the decrease in leptin levels may be attributed to reduced adipose tissue due to adrenalectomy. On the other hand, the decreases in glucose and triglyceride levels might be the consequence of reduced lipogenesis and impaired gluconeogenesis with the effect of adrenalectomy. It was concluded that adrenalectomy might prevent obesity by affecting leptin and intermediate metabolism.

Key words leptin; adrenalectomy; diet-induced obesity

Obesity, which affects up to $35 \%$ adult population of developed countries, is associated with serious comorbidities, including a high incidence of type 2 diabetes, cardiovascular disease, osteoarthritis, and an increased risk of many forms of cancer. ${ }^{1)}$ To explain the etiology of obesity, many factors have been suggested. The lipostasis theory postulated that the size of the body fat depots is sensed by the central nervous system through a product of fat metabolism, which circulates in the blood and affects energy balance by influencing the hypothalamus (Kennedy et al., 1953). ${ }^{2)}$ This substance was later called as leptin. Leptin, a 167-amino acid protein, is expressed primarily by adipocytes regulates food intake, which was initially identified by Zhang and colleagues in 1994. ${ }^{3)}$

Plasma leptin levels and ob gene expression are correlated with adipose tissue mass, suggesting that adipose depot size is a major regulator of leptin production. Little is known about the nutritional regulation of leptin production. Restriction and refeeding are known to regulate plasma leptin and ob gene expression in rodents and humans. The effect of high-fat diet on circulating leptin levels has been studied in several animal models. A high-fat diet also increased ob gene expression in adipose tissue of male Sprague-Dawley rats. However, this does not prevent hyperphagia and obesity, suggesting that high-fat feeding makes rodents resistant to leptin. $\left.{ }^{4}\right)$

The role of glucocorticoids in pathogenesis of obesity has been investigated. High levels of glucocorticoids have been shown in many genetically obese rats. High doses of glucocorticoids have also been shown to induce leptin gene expression when injected into rats. ${ }^{5,6)}$ This was accompanied by body weight lose. Adrenalectomy and glucocorticoid antagonists have impaired obesity in ob/ob, $\mathrm{db} / \mathrm{db}$ and some obese mice. Low dose glucocorticoid replacement also restored obese phenotype. ${ }^{7)}$ On the basis of these reports, we also aimed to investigate how adrenalectomy (AD) affects leptin levels in rats with diet-induced obesity.

\section{MATERIALS AND METHODS}

Male Sprague-Dawley rats $(160-180 \mathrm{~g}$ body wt) were included in the study. Rats were housed in a room under conditions of controlled temperature (22 \pm 1$)$, illumination $(8: 00$ a.m. $-8: 00$ p.m.), and humidity in six groups for 10 weeks. Before the experimental procedure, they were assigned to one of two dietary groups: one having a high-calorie diet (OB, $n=19$ ), one having a standard laboratory diet (LE, $n=16$ ). Each group were also subgroupped into three (Table 1). The high-calorie diet consisted of $33 \%$ powdered laboratory diet, $33 \%$ condensed milk, and sucrose by weight, with the remainder being water; this provided $68 \%$ energy as carbohydrate, $17 \%$ as protein, and $13 \%$ as fat. The standard laboratory diet provided $65 \%$ energy as carbohydrate, $24 \%$ as protein and $9 \%$ as fat. ${ }^{8}$ Body weights were monitored weekly. Eight weeks later, rats were anesthetized with thiopental $(25 \mathrm{mg} / \mathrm{kg})$, and ten animals in the obese group (OBAD) and eight animals in the lean group (LEAD) were adrenalectomized through dorsal incisions. Four animals from the obese (OBSHAM) and four from the lean dietary

Table 1. Groups and Subjects

\begin{tabular}{lcc}
\hline \multicolumn{1}{c}{ Groups } & Obese (OB) & Lean (LE) \\
\hline Adrenalectomy (AD) & 10 & 8 \\
Sham operation (SHAM) & 4 & 4 \\
Control (CON) & 5 & 4 \\
Total & 19 & 16 \\
\hline
\end{tabular}


groups (LESHAM) received sham operations. Five animals in the obese group and four animals in the lean group were assigned as controls. After surgery, they were given their respective diets for an additional two weeks. In addition, adrenalectomized rats received $0.9 \% \mathrm{NaCl}$ solution. Two weeks later, the blood samples were taken from the heart folloving anesthesia and used for serum leptin, glucose, triglycerides and cholesterol determinations. Serum leptin levels were determined with RIA. Glucose determination was performed with hexokinase method, and triglyceride and cholesterol levels with peroxidase method in Hitachi 717 autoanalyzer.

Statistical Analysis Two way analysis of variance (ANOVA) was used to determine the effects of diet, surgery, and diet-surgery interaction (Statistica package program). Least Significant Difference (LSD) test was used to compare the differences of measures between the groups and within the same group. Differences were considered significant at $p<0.05$.

\section{RESULTS}

High-calorie feeding resulted in a body weight increase of about $20 \%$ and in the development of obesity for a period of 10 week $(p<0.001)$. The weights $(\mathrm{g})$ of the rats before adrenalectomy (at the 8th week) were $321 \pm 21.44$ in OBAD, $329 \pm 19.17$ in OBSHAM, $342 \pm 34.92$ in OBCON, 256.5 \pm 29.63 in LEAD, 269 \pm 21.69 in LESHAM, and 258.5 \pm 21.14 in LECON. Adrenalectomy caused weight loss in both lean and obese animals but the differences did not reach to a statistically significant level. However, in the control groups there were a continuous increase in the weights until the 10th

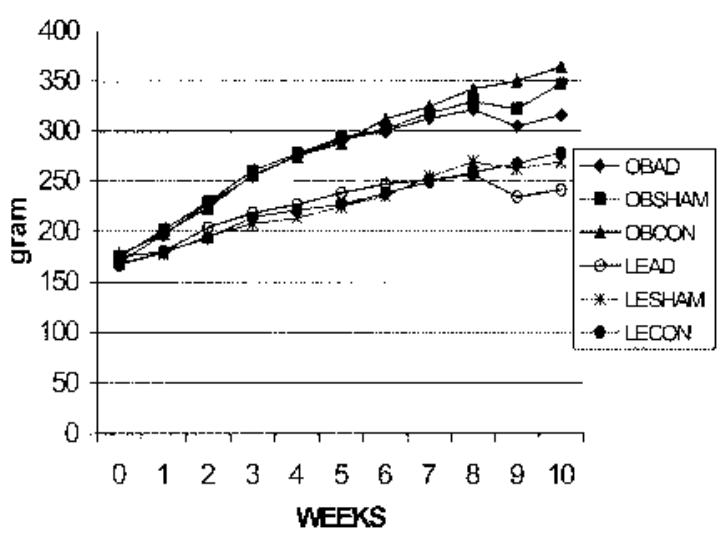

Fig. 1. The Changes in Weights of the Animals for Ten-Week Period week. In the SHAM groups, although there was a weight decrease after sham operation (at the 9th week), weight gain was observed at the 10th week. Contrarily, in AD groups after operation, the animals could not reach to the weights at the 8th week (Fig. 1). Table 2 summarizes means and standard deviations of the parameters.

Leptin levels in obese rats were higher than those of lean ones $(p<0.001)$, (Tables 3,4$)$. Glucose $(p<0.05)$ and triglyceride $(p<0.01)$ concentrations also increased in obese rats compared to lean rats (Tables 3, 4). Adrenalectomy also decreased leptin $(p<0.001)$ and triglyceride $(p<0.01)$ concentrations in both obese and lean animals compared with control groups (Tables 4, 5).

\section{DISCUSSION}

Results from the present experiment showed that highcalorie feeding resulted in increases in body weight and in serum leptin levels of rats. At the end of 10th week of the high-calorie diet, rats had significantly increased body weights, eventually being $20 \%$ heavier than control rats $(p<0.001)$, which was in agreement with previous reports that showed that feeding the high-calorie diet developed obesity in several rodent models. ${ }^{9-11)}$ Feeding with diet which has high lipid proportion has developed obesity and adipose tissue mass increase. Therefore, leptin levels also have shown elevations. ${ }^{12)}$

Glucocorticoids stimulate leptin production via increased ob gene expression. ${ }^{13)}$ In addition, exogenous administration of glucocorticoids results in hyperinsulinemia ${ }^{14)} \beta$-cell hyperplasia, and insulin resistance. ${ }^{15)}$ Adrenalectomy has decreased weight gain, ${ }^{16)}$ food intake and fat deposition in rodents. ${ }^{17)}$ Moreover, adrenalectomy has been shown to have a number of effects, including increasing energy expenditure due to enhanced thermogenesis, abolishing insulin resistance in muscle, and lowering plasma levels of insulin, glucose, and triglycerides. ${ }^{18,19)}$

The present study showed that adrenalectomy attenuated

Table 3. Summary of Variance Analysis

\begin{tabular}{lllc}
\hline \hline Parameters & $\begin{array}{c}\text { Effect of diet } \\
\text { (p) (OB-LE) }\end{array}$ & $\begin{array}{c}\text { Effect of operation } \\
\text { (p) (AD-CON) }\end{array}$ & $\begin{array}{c}\text { Interaction } \\
\text { (diet vs. operation) (p) }\end{array}$ \\
\hline Leptin & $0.0001^{* * *}$ & $0.00046^{* * *}$ & 0.98 \\
Glucose & $0.034^{*}$ & 0.165 & 0.69 \\
Triglycerides & $0.005^{* *}$ & $0.048^{*}$ & 0.86 \\
Cholesterol & 0.564 & 0.242 & 0.83 \\
\hline
\end{tabular}

$* p<0.05 ; * * p<0.01 ; * * * p<0.001$.

Table 2. The Mean and Standard Deviation Values of the Groups

\begin{tabular}{lccccc}
\hline \hline & \multicolumn{3}{c}{ Parameters } \\
\cline { 2 - 5 } Groups & $\begin{array}{c}\text { Body weight }(\mathrm{g}) \\
\text { X S.D. }\end{array}$ & $\begin{array}{c}\text { Leptin }(\mathrm{ng} / \mathrm{ml}) \\
X \pm \text { S.D. }\end{array}$ & $\begin{array}{c}\text { Glucose }(\mathrm{mg} / \mathrm{dl}) \\
X \pm \text { S.D. }\end{array}$ & $\begin{array}{c}\text { Triglycerides }(\mathrm{mg} / \mathrm{dl}) \\
X \pm \text { S.D. }\end{array}$ & $\begin{array}{c}\text { Cholesterol (mg/dl) } \\
X \pm \text { S.D. }\end{array}$ \\
\hline OB+AD $(n=10)$ & $315.5 \pm 6.08$ & $1.93 \pm 0.29$ & $125.30 \pm 22.26$ & $103.20 \pm 21.21$ & $56.90 \pm 10.30$ \\
OB+SHAM $(n=4)$ & $347 \pm 5.29$ & $2.22 \pm 0.20$ & $142.25 \pm 16.46$ & $149.75 \pm 22.43$ & $62.25 \pm 8.46$ \\
OB+CON $(n=5)$ & $364 \pm 37.65$ & $2.27 \pm 0.21$ & $141.00 \pm 18.43$ & $148.00 \pm 20.17$ & $56.00 \pm 5.92$ \\
LE+AD $(n=8)$ & $241 \pm 25.32$ & $0.93 \pm 0.08$ & $110.30 \pm 18.79$ & $64.13 \pm 11.63$ & $52.50 \pm 7.23$ \\
LE+SHAM $(n=4)$ & $269 \pm 21.70$ & $1.24 \pm 0.15$ & $128.25 \pm 12.01$ & $103.75 \pm 17.59$ & $60.75 \pm 14.45$ \\
LE+CON $(n=4)$ & $278 \pm 18.06$ & $1.33 \pm 0.21$ & $126.50 \pm 13.40$ & $99.50 \pm 16.78$ & $56.00 \pm 8.60$ \\
\end{tabular}


Table 4. The Differences in Obese and Lean Groups

\begin{tabular}{|c|c|c|c|}
\hline Parameters/groups & OBAD-LEAD & OBSHAM-LESHAM & OBCON-LECON \\
\hline Body weights (g) & $74.5^{* * *}$ & $78 * * *$ & $86^{* *}$ \\
\hline Leptin $(\mathrm{ng} / \mathrm{ml})$ & $1 * * *$ & $0.98 * * *$ & $0.94 * * *$ \\
\hline Glucose (mg/dl) & 15.00 & 14 & 14.5 \\
\hline Triglycerides (mg/dl) & $39.07 * *$ & $46^{* *}$ & $48.5^{* *}$ \\
\hline Cholesterol (mg/dl) & 4.4 & 1.50 & 0 \\
\hline
\end{tabular}

${ }^{*} p<0.05 ; * * p<0.01 ; * * * p<0.001$.

Table 5. The Statistical Evaluation of the Differences in Subgroups

\begin{tabular}{|c|c|c|c|c|c|}
\hline \multirow{2}{*}{ The difference between } & \multicolumn{5}{|c|}{ Parameters } \\
\hline & Body weights (g) & Leptin (ng/ml) & Glucose $(\mathrm{mg} / \mathrm{dl})$ & Triglycerides (mg/dl) & Cholesterol (mg/dl) \\
\hline OBAD-OBSHAM & -31.5 & $-0.29 *$ & -16.95 & $-46.55^{* *}$ & -5.35 \\
\hline OBAD-OBCON & $-48.5^{*}$ & $-0.34 * *$ & -15.7 & $-44.8^{* *}$ & 0.90 \\
\hline OBSHAM-OBCON & -17 & -0.05 & 1.25 & 1.75 & 6.25 \\
\hline LEAD-LESHAM & -28 & $-0.31^{*}$ & -17.95 & $-39.62 * *$ & -8.25 \\
\hline LEAD-LECON & $-37^{*}$ & $-0.40 * *$ & -16.20 & $-35.37 * *$ & -3.50 \\
\hline LESHAM-LECON & -9 & -0.09 & 1.75 & 4.25 & -4.75 \\
\hline
\end{tabular}

$* p<0.05 ; * * p<0.01 ; * * * p<0.001$.

body weight gain in both lean and obese rats, ${ }^{16,21,22)}$ similarly to previous studies.

In the present study, serum leptin levels were high in obese rats compared with lean rats $(p<0.001)$. This may be explained by the fact that obese rats have very high adipose tissue compared with lean ones, which may be due to adrenalectomy, decreasing adipose tissue mass. Besides, sham operation failed to decrease leptin levels in obese and lean rats.

Similarly, Widdowson and colleagues have showed that obese rats have high levels of leptin compared with lean rats. The increase in leptin levels has been explained with increase in body mass. ${ }^{8}$ A novel finding was that adrenalectomy per se reduced ob mRNA levels in white adipose tissue by fivefold, possibly due to the lack of circulating glucocorticoids, which have been shown to enhance ob expression both in vivo and in vitro. ${ }^{6}$ ) Adrenalectomy markedly diminished the increase in ob expression seen after intracerebroventricular neropeptid Y (ICV NPY, a potent stimulator of food intake) infusion, although NPY did tend to increase the low ob mRNA levels of adrenalactomized rats toward normal control values. ${ }^{7)}$ Earlier studies showed that adrenalectomy decreased weight gain, lipid intake and adipose tissue mass in rodents. Glucocorticoid replacement prevented the effects of AD on weight gain and body fat deposition. ${ }^{17,20-22)}$

Circulating glucose and triglyceride levels have increased in obese rats due to impaired metabolism. In the present study, circulating glucose $(p<0.05)$ and triglyceride $(p<0.01)$ levels have significantly increased in obese rats compared with lean ones. Glucose levels in OBAD rats lower than OBCON and OBSHAM groups, but its differencies were not statistically significant. This may be due to the absence of glucocorticoid stimulation because, the lack of glucocorticoids have decreased appetite, lipogenesis and gluconeogenesis.

Similarly to the recent studies, ${ }^{17,20,21)}$ AD significantly reduced trigliyceride levels in lean rats $(p<0.01)$. AD reduced glucose levels in lean rats, but the reduction was not statistically significant.
As a result, $\mathrm{AD}$ resulted in weight lost, and in reduced leptin and triglyceride levels in diet-induced obese SpragueDawley rats. Conclusions in studies with several models of obesity have shown that leptin levels are high in obese animals and leptin has decreased adipose tissue mass. However, there are some differences in the explanation of some parameters. Although the diet-induced-obese rats have increased plasma leptin levels, several studies have been performed associated with leptin gene, receptor, post-receptor defects and leptin transport through blood-brain barrier in order to explain why the obesity is not prevented. However the explanations are poorly illuminated. Diet-induced obesity in rodents is more similarly to the obesity in human than other available models. Nevertheless, in order to be explained better the association between leptin, AD, glucocorticoids, NPY and other metabolic parameters; leptin, glucocorticoids, NPY, insulin, hormon sensitive-lipase, and associated analytes should be analyzed in a detailed manner in appropriate model, in body fluid and tissues, and in gene levels.

\section{REFERENCES}

1) Van Heek M., Compton D. S., France C. F., J. Clin. Invest., 99, 385390, (1997).

2) Caro J. F., Sinha M. K., Kolaczynski J. W., Zhang P. L., Considine R. V., Diabetes, 45, 1455-1462 (1996).

3) Zhang Y., Proenca R., Maffei M., Barone M., Leopold L., Friedman M. J., Nature (London), 372, 425-431 (1994).

4) Kabir M., Guerre-Millo M., Laromiguiere M., Metabolism, 49, 764769 (2000).

5) Hamman A., Matthaei S., Exp. Clin. Endocrinol. Diabetes, 104, $293-$ 300 (1996).

6) De Vos P., Salasin R., Auwerx J., Staels B., J. Biol. Chem., 270, 15958-15961 (1995).

7) Sainsbury A., Cusin I., Jeanrenaud R. F., Jeanrenaud B., Diabetes, 46, 209-214 (1997).

8) Widdowson P. S., Upton R., Buckingham R., Arch J., Williams G., Diabetes, 46, 1782-1785 (1997).

9) Levin B. E., Sullivan A. C., Am. J. Physiol., 253, R475-481 (1987).

10) West D. B., Boozer C. N., Moody D. L., Atkinson R. L., Am. J. Physiol., 262, R1025-R1032 (1992). 
11) Arvaniti K., Deshaies Y., Richard D., Am. J. Physiol., 275, R105R111 (1998).

12) Hamman A., Matthaei S., Exp. Clin. Endocrinol. Diabetes, 104, $293-$ 300 (1996).

13) Tataranni P. A., Pratley R., Maffei M., Ravussin E., Internat. J. Obesity, 21, 327-330 (1997).

14) Plotsky P. M., Thrivikraman K. V., Watts A. G., Hauger R. L., Endocrinology, 130, 1931-1941 (1992).

15) Bornstein S. R., Uhlmann K., Haidan A., Bornstein M. E., Scherbaum W. A., Diabetes, 46, 1235-1238 (1997).

16) Bray G. A., Stern J. S., Castonguay T. W., Am. J. Physiol., 262, E32E39 (1992).
17) Trocki O., Baer D. J., Castonguay T. W., Am. J. Physiol., 269, R708R719 (1995).

18) Saito M., Bray G. A., Am. J. Physiol., 246, R20-R25 (1984).

19) Yukimura Y., Bray G. A., Wolfsen A. R., Endocrinology, 103, 1924 1928 (1978).

20) Freedman R. M., Hortwitz A. B., Stern S. J., Am. J. Physiol., 250, R595-R607 (1986).

21) Costanguay T. W., Dallman M. F., Stern J. S., Am. J. Physiol., 251, R923-R933 (1986).

22) Freedman M. R., Castonguay T. W., Stern J. S., Am. J. Physiol., 249, R584-R594 (1985). 